\title{
Niveles referenciales de nutrientes en la solución del suelo para el diagnóstico nutricional en el cultivo protegido del tomate
}

\author{
Nutrient levels of reference in the soil solution to the nutrition \\ diagnostic in the tomato protected crop
}

\author{
María Isabel Hernández Díaz ${ }^{*}$, Marisa Chailloux Laffita, Víctor Moreno Placeres, \\ Alberto Igarza Sánchez, Anselma Ojeda Veloz
}

\section{RESUMEN}

La producción de hortalizas en sistemas protegidos implica una explotación muy intensiva del cultivo, por tales motivos se requiere del monitoreo continuo para maximizar su nutrición y rendimiento, más aun cuando su explotación se realiza en suelo, pues se desconoce la magnitud de las interacciones que ocurren entre la solución nutritiva y el suelo mismo. El estudio se realizó en el Instituto de Investigaciones Hortícolas "Liliana Dimitrova" (Mayabeque, Cuba) en un suelo ferralítico rojo típico en invierno y primavera-verano. Se utilizó el híbrido comercial HAZERA 3019. Se realizó la caracterización de la solución fertilizante y de la solución del suelo, para esta última se colocaron en el interior de la instalación ocho sondas de succión. Se determinó el pH, la concentración de $\mathrm{NO}_{3}^{-}, \mathrm{K}^{+}, \mathrm{Ca}^{++}$y $\mathrm{Mg}^{++}$y la conductividad eléctrica (CE). Se cuantificó la masa seca total, la absorción de $\mathrm{N}$, $\mathrm{P}, \mathrm{K}, \mathrm{Ca}$ y $\mathrm{Mg}$ y el rendimiento. Las variables de cultivo evaluadas durante el invierno fueron estadísticamente superiores a las cuantificadas en primavera-verano. La concentración de nutrientes en sondas de succión varió en dependencia de la época, la fase del cultivo y las características del elemento. La alta movilidad del $\mathrm{NO}_{3}{ }^{-}$y del $\mathrm{K}^{+}$y la presencia de indicadores productivos y de crecimiento superiores en invierno condicionaron su disminución con relación a la solución fertilizante, mientras que el $\mathrm{Ca}^{++}$ y el $\mathrm{Mg}^{++}$se acumularon en el suelo. Las concentraciones de $\mathrm{NO}_{3}{ }^{-} \mathrm{K}^{+}, \mathrm{Ca}^{++} \mathrm{y} \mathrm{Mg}^{++}$en sondas de succión en primavera-verano estuvieron en un rango de 5,99-9,06, 3,16-7,68, 2,91-4,27 y 1,34-2,14 meq/L, respectivamente. En invierno variaron entre 6,87$11,47,2,89-8,83,2,81-5,60$ y 1,04-2,31 meq/L. La variación máxima obtenida para la CE en sondas de succión fue de 0,50 mS/ cm con relación a la solución fertilizante.

Palabras clave: Solanum lycopersicum L., fertirriego, sondas de succión, conductividad eléctrica, solución nutritiva.

\begin{abstract}
The production of horticultural crop in protected systems implies a very intensive exploitation of the cultivation, for what is required of the continuous supervise to maximize its nutrition and yield, more even when its exploitation is carried out in soil, because the magnitude of the interactions that happen between the nutritious solution and the same soil is ignored. The study was carried out in the "Liliana Dimitrova" Horticultural Research (Mayabeque, Cuba) in a typical Red Ferralitic soil in winter and spring-summer. The hybrid commercial HAZERA 3019 was used. It was make the characterization of the solution fertilizer and soil solution, for this last one it placed inside the installation eight suction cups. It was determined the $\mathrm{pH}$, the $\mathrm{NO}_{3}^{-}, \mathrm{K}^{+}, \mathrm{Ca}^{++}$and $\mathrm{Mg}^{++}$concentration and the electric conductivity (EC). It was quantified the total dry mater, the absorption of $\mathrm{N}, \mathrm{P}, \mathrm{K}, \mathrm{Ca}$ and $\mathrm{Mg}$ and the yield. The cultivation variables evaluated during the winter went superiors statistically to the quantified in spring-summer. The concentration of nutrients in suction cups varied in dependence of the plantation time, the cultivation phase and the element characteristics. The high $\mathrm{NO}_{3}{ }^{-}$and $\mathrm{K}^{+}$mobility and the presence of superiors indicators productive and growth in winter conditioned their decrease with relationship to the solution fertilizer, while the $\mathrm{Ca}^{++}$and $\mathrm{Mg}^{++}$was accumulated in the soil. The concentrations of $\mathrm{NO}_{3}^{-}, \mathrm{K}^{+}$, $\mathrm{Ca}^{++}$and $\mathrm{Mg}^{++}$in suction cups in spring-summer were respectively in a range of 5.99-9.06, 3.16-7.68, 2.91-4.27 and 1.34-2.14 meq/L. In winter they varied among 6.87-11.47, 2.89-8.83, 2.81-5.60 and 1.04-2.31 meq/L. The maximum variation obtained for the CE in suction cups was of $0.50 \mathrm{mS} / \mathrm{cm}$ with relationship to the fertilizer solution.
\end{abstract}

Key words: Solanum lycopersicum $L$., fertirrigation, suction cups, electric conductivity, nutrient solution.

\footnotetext{
1 Instituto de Investigaciones Hortícolas "Liliana Dimitrova", Km 33 1⁄2 carretera Quivicán Bejucal, Municipio Quivicán, Provincia Mayabeque, Cuba.

* Autor para correspondencia: nutricion1@liliana.co.cu
}

Fecha de Recepción: 14 Agosto 2013.

Fecha de Aceptación: 27 Febrero 2014. 


\section{Introducción}

Para diseñar planes de fertirrigación que se ajusten a una determinada condición edafoclimática se debe disponer de suficiente información que permita identificar alteraciones en el estado nutricional de las plantas, a partir del monitoreo de los factores que afectan el sistema suelo-plantaagua (Voogt, 2006).

El cultivo protegido en suelo constituye un ambiente complejo, pues cuando una determinada dosis de nutriente se suministra a la plantación es muy difícil de estimar la proporción de ella que pasa a la CIC. Por tales motivos, el análisis de la solución del suelo (SS) constituye el intermediario nutritivo entre el complejo absorbente y la planta y permite conocer la interacción que se produce entre la solución fertilizante y el sustrato (Cano y Rojo, 2004; Murillo et al., 2006). El análisis de la SS se logra a partir de la colocación de las sondas de succión que permiten cuantificar, in situ, la composición química de la solución en contacto con las raíces (Salas et al., 2009).

El estudio de la solución de suelo permite establecer el índice de demanda de nutrientes básicos, de manera que su aporte en la solución fertilizante real y el contenido en la solución de suelo a diferentes profundidades nos ofrezca una orientación de la demanda y disponibilidad de estos nutrientes. Asimismo, también indica de manera aproximada el índice de lixiviación de un determinado nutriente y los riesgos de contaminación, como es el caso de los fertilizantes nitrogenados para optimizar el manejo de la fertilización nitrogenada (Granados et al., 2013).

En cuanto a los niveles de referencia para establecer una adecuada composición de la solución del suelo, Romo (2006) afirma que el material genético, las características del agua de riego y su variación en el ciclo son factores que dificultan identificar indicadores reales y aplicables a cualquier condición edafoclimática. También se considera que la profundidad del suelo, la distribución de los nutrientes antes y después del fertirriego, su evolución durante el ciclo de crecimiento y la situación dentro del invernadero pueden modificar la solución en contacto con las raíces (Lao et al., 2003).

En Cuba, desde el punto de vista científico, se identifica como dificultad la insuficiente información sobre indicadores de referencia de concentración nutrimental en la solución del suelo y su relación con la solución fertilizante que permitan elevar la eficiencia de la nutrición en el cultivo protegido del tomate y caracterizar, de manera correcta, la solución en torno a la raíz, para las variadas condiciones de suelo y clima existentes. Implantar un adecuado seguimiento nutricional supone tener un mayor control del medio, una mayor eficiencia en la producción, mayor calidad de nuestras cosechas y menor contaminación del medio. Basado en lo anterior, el presente estudio tuvo como objetivo establecer valores orientativos de concentración de nutrientes en la solución del suelo con relación a la solución fertilizante para ser utilizados en el seguimiento del fertirriego en el cultivo protegido del tomate.

\section{Materiales y Métodos}

El presente estudio se desarrolló en el Instituto de Investigaciones Hortícolas "Liliana Dimitrova", en el Municipio de Quivicán, provincia de Mayabeque, Cuba, a $22^{\circ} 52^{\prime} \mathrm{N}$ y $82^{\circ} 23^{\prime} \mathrm{W}$ y a una altura sobre el nivel del mar de $68 \mathrm{~m}$ en condiciones de cultivo protegido (instalación de $540 \mathrm{~m}^{2}, 4$ de altura, rafia plastificada en la parte superior y malla sombreadora por los laterales y el frente). Se utilizó el híbrido de tomate Israelí HAZERA 3019 (HA 3019) de crecimiento determinado, con un ciclo entre 100 y 120 días y un rendimiento promedio para el trópico cubano entre 60 y 80 t/ha; posee además alta resistencia al virus del encrespamiento amarillo de la hoja de tomate o TYCLV (Casanova et al., 2007). La caracterización de la solución del suelo (SS) y de la solución fertilizante (SF) se realizó en dos épocas de plantación; primavera-verano (marzo-julio/2009) e invierno (septiembre-diciembre de 2009).

El cultivo se estableció en un suelo ferralítico rojo típico, o Ferralsol Rhodic en correlación con el World Reference Base for Soil Resources (Hernández et al., 2000). Se caracteriza por poseer un $\mathrm{pH}$ ligeramente alcalino (7,75 por potenciometría), altos contenidos de fósforo $(66,44 \mathrm{mg} / 100 \mathrm{~g}$ por Oniani) y potasio $(62,87 \mathrm{mg} / 100 \mathrm{~g}$ por Oniani), materia orgánica media (2,30\% por Walkey-Black) e inadecuada relación $\mathrm{K}^{++} / \mathrm{Ca}^{++}+\mathrm{Mg}^{++}(0,10)$. Las soluciones nutritivas que recibió el cultivo del tomate en las dos épocas de estudio, según lo recomendado por Hernández et al. (2009), aparecen en la Tabla 1. Para evitar efectos antagónicos entre los cationes se varió la concentración de $\mathrm{Ca}^{++} \mathrm{y} \mathrm{Mg}^{++}$en función 
Tabla 1. Concentración de nutrientes $(\mathrm{mg} / \mathrm{L})$ en la solución fertilizante para dos épocas de plantación en el cultivo protegido del tomate (híbrido HA 3019).

\begin{tabular}{|c|c|c|c|c|c|c|c|c|c|c|c|c|}
\hline \multirow{2}{*}{ Fases $^{1}$} & \multicolumn{6}{|c|}{ Época primavera-verano } & \multicolumn{6}{|c|}{ Época de invierno } \\
\hline & $\mathrm{N}$ & $\mathrm{P}_{2} \mathrm{O}_{5}$ & $\mathrm{~K}_{2} \mathrm{O}$ & $\mathrm{CaO}$ & $\mathrm{MgO}$ & $\mathrm{N}: \mathrm{K}$ & $\mathrm{N}$ & $\mathrm{P}_{2} \mathrm{O}_{5}$ & $\mathrm{~K}_{2} \mathrm{O}$ & $\mathrm{CaO}$ & $\mathrm{MgO}$ & $\mathrm{N}: \mathrm{K}$ \\
\hline I & 0 & 152 & 0 & 0 & 0 & - & 0 & 152 & 0 & 0 & 0 & - \\
\hline II & 90 & 95 & 90 & 111 & 26 & $1: 1$ & 108 & 114 & 108 & 134 & 32 & $1: 1$ \\
\hline III y V & 134 & 95 & 267 & 159 & 38 & $1: 2.5$ & 161 & 114 & 400 & 239 & 57 & $1: 2.0$ \\
\hline IV & 155 & 95 & 313 & 187 & 44 & $1: 2.5$ & 189 & 114 & 475 & 284 & 68 & $1: 2.0$ \\
\hline
\end{tabular}

${ }^{1}$ Fase I : trasplante-emisión del primer racimo floral. Fase II: emisión del primer racimo floral-cuaje del tercer racimo. Fase III: cuaje del tercer racimo-inicio de cosecha. Fase IV: inicio de cosecha-plena producción. Fase V: plena producción-final de la plantación.

Tabla 2. Variables de clima para dos épocas de plantación en el cultivo protegido del tomate.

\begin{tabular}{lllll}
\hline \multirow{2}{*}{ Época } & \multicolumn{3}{c}{ Temperatura $\left({ }^{\circ} \mathrm{C}\right)$} & \multirow{2}{*}{ Humedad relativa (\%) } \\
\cline { 2 - 4 } & Máxima & Mínima & Media & 72,77 \\
\hline Primavera-verano & 36,575 & 24,36 & 30,7 & 66,325 \\
Invierno & 30,31 & 17,645 & 23,975 & \\
\hline
\end{tabular}

de la del $\mathrm{K}^{+}$, manteniendo una relación $\mathrm{K}_{2} \mathrm{O} / \mathrm{CaO}$ $+\mathrm{MgO}$ de 1,36.

En la Tabla 2 se presentan los valores promedio de temperatura y humedad relativa para cada época de estudio.

Se utilizó un sistema de riego por goteo, con mangueras de PVC negro de $16 \mathrm{~mm}$, goteros a $0,45 \mathrm{~cm}$ y con una entrega de 2,50 L/hora. Para la programación del riego se ubicó un tensiómetro (medidor del potencial matricial del suelo en un rango de 0 a $100 \mathrm{kPa}$ modelo IROMETER) en el centro de la instalación a una profundidad de $20 \mathrm{~cm}$ y a una distancia de $10 \mathrm{~cm}$ de la planta y del emisor. El riego se efectuó cuando la lectura fue superior a $15 \mathrm{Kpa}$. Según las Normas Riverside, el agua de riego se clasifica como $\mathrm{C}_{2} \mathrm{~S}_{1}$ (salinidad media, bajo contenido de $\mathrm{Na}^{+}$y apta para el riego) (Cadahía, 2005).

El cálculo de los aportes de fertilizantes (g/ m3) se efectuó teniendo en cuenta la concentración de $\mathrm{HCO}_{3}^{-}, \mathrm{K}^{+}, \mathrm{Ca}^{++}$y $\mathrm{Mg}^{++}$en el agua de riego y el balance de las soluciones nutritivas comenzó con la neutralización de los iones $\mathrm{HCO}_{3}^{-}$hasta garantizar una concentración de $0,50 \mathrm{mmol} / \mathrm{L}$. Se utilizó una bomba inyectora, modelo AMIAD a razón de $2 \mathrm{~L} / \mathrm{min}$. Se utilizaron los siguientes portadores: $\mathrm{H}_{3} \mathrm{PO}_{4}\left(1040,00 \mathrm{~g} \mathrm{P}_{2} \mathrm{O}_{5} / \mathrm{L}\right), \mathrm{HNO}_{3}$ $(145,30 \mathrm{~g} \mathrm{~N} / \mathrm{L}), \mathrm{NH}_{4} \mathrm{NO}_{3}(34,80 \%$ de N, $17,50 \%$ de $\mathrm{N}$ nítrico y $17,30 \%$ amoniacal), $\mathrm{KNO}_{3}(12 \%$ de $\mathrm{N}$ nítrico, $45 \%$ de $\mathrm{K}_{2} \mathrm{O}$ y $1,20 \%$ de $\left.\mathrm{SO}_{4}\right), \mathrm{Ca}\left(\mathrm{NO}_{3}\right)_{2}$ (26\% de $\mathrm{CaO}, 14,50 \%$ de $\mathrm{N}$ nítrico y $1,00 \%$ de $\mathrm{N}$ amoniacal), $\mathrm{K}_{2} \mathrm{SO}_{4}\left(50 \%\right.$ de $\mathrm{K}_{2} \mathrm{O}$ y $54 \%$ de $\mathrm{SO} 4$ ), $\mathrm{MgSO}_{4}\left(16 \%\right.$ de $\mathrm{MgO}$ y $39 \%$ de $\mathrm{SO}_{4}$ ) y Premiun Quelato $(0,05 \%$ de B, $0,05 \%$ de $\mathrm{Cu}, 4,00 \%$ de $\mathrm{Fe}$, $2,00 \%$ de Mn, $0,01 \%$ de Mo y 1,00\% de Zn) a razón de $50 \mathrm{~g} / \mathrm{m}^{3}$, una vez por semana.

Durante el desarrollo del cultivo se cuantificó la masa seca total $\left(\mathrm{g} / \mathrm{m}^{2}\right)$ al final de las fases II, III y IV del ciclo del cultivo. Se extrajeron 20 plantas al azar, se secaron en una estufa a $65^{\circ} \mathrm{C}$ hasta peso constante y la masa seca se determinó en balanza técnica. Se cuantificó además la absorción de N, P, $\mathrm{K}$, Ca y $\mathrm{Mg}\left(\mathrm{g} / \mathrm{m}^{2}\right)$, estos análisis se realizaron en las plantas provenientes de la evaluación de masa seca. La absorción se calculó a partir de la siguiente fórmula: Q = B x EMS/100, donde Q: absorción $\left(\mathrm{g} / \mathrm{m}^{2}\right)$, B: Biomasa $\left(\mathrm{g} / \mathrm{m}^{2}\right)$ y EMS: porcentaje del elemento en materia seca (\%).

Se efectuaron entre 9 y 12 cosechas y el período de recolección tuvo una duración de 46 días para la época primavera-verano y de 56 días para la época de invierno. Se determinó la masa promedio de un fruto $(\mathrm{g})$ y el rendimiento individual en las categorías de calidad extra (diámetro ecuatorial del fruto $>75 \mathrm{~mm}$ ), primera (entre 65-74 $\mathrm{mm}$ ), extra + primera $(\mathrm{E}+\mathrm{P})$ y segunda (entre 55-64 $\mathrm{mm})(\mathrm{Cuba}$, MINAG, 2010), mientras que el rendimiento total (t/ha) se calculó sobre la base de la masa de todos los frutos por parcela.

Con el objetivo de colectar la solución fertilizante (SF) emitida durante todo el fertirriego se colocaron 
recipientes debajo del gotero, en cuatro puntos del área experimental (réplicas). Este muestreo se realizó a partir de la fase II del ciclo del cultivo y una vez en las fases III y IV. Para la caracterización de la solución del suelo (SS) se ubicaron ocho sondas de succión en el interior de la instalación, a $10 \mathrm{~cm}$ de la planta y a $20 \mathrm{~cm}$ de profundidad, se efectuó un vacío dos horas después del riego con un vacuómetro a una presión de 75 centibares y la muestra se extrajo al día siguiente con una jeringuilla propia del equipo. Esta operación se realizó tres veces por semana. Se determinó, tanto en la SF como en la $\mathrm{SS}$, la conductividad eléctrica $(\mathrm{mS} / \mathrm{cm})$, el $\mathrm{pH}$ y la concentración de $\mathrm{NO}_{3}^{-}, \mathrm{K}^{+}, \mathrm{Ca}^{++} \mathrm{y} \mathrm{Mg}^{++}(\mathrm{mmol} / \mathrm{L})$.

Se realizó un Análisis de Varianza de clasificación doble para las variables evaluadas en la planta. Se comprobó la normalidad mediante los estadígrafos de asimetría y de curtosis, así como la homogeneidad de varianza con la dócima de Bartlett. Las medias se compararon mediante la prueba de Tukey con un nivel de significación de 0,05 . Se utilizó el programa SPSS versión 11.5.

\section{Resultados y Discusión}

La masa seca total cuantificada al final de las fases II, III y IV del ciclo del tomate durante la época de invierno fue significativamente superior a la encontrada para la época primavera-verano. De igual forma sucedió con la absorción de N, K, Ca y Mg, determinada al final de la fase IV (Tabla 3).
El rendimiento y la distribución por calidades decrecen también en la época de primaveraverano con relación al invierno, con reducciones significativas en el rendimiento $\mathrm{E}+\mathrm{P}$ y total de $29,43 \%$ y $22,76 \%$, respectivamente. Los rendimientos obtenidos en ambas épocas son característicos de la época de estudio y del cultivar utilizado, 50-60 $\mathrm{t} / \mathrm{ha}$ en primavera verano y $80-100 \mathrm{t} / \mathrm{ha}$ en invierno (Casanova et al., 2007).

La época de invierno se caracterizó por presentar valores de temperatura más favorables para el cultivo del tomate, principalmente durante las fases de llenado del fruto y de cosecha (a partir de la fase III). Lo anterior incidió en que las plantas de tomate acumularan una mayor cantidad de biomasa durante la época de invierno, existiera una eficiencia superior en el proceso de fotosíntesis y como consecuencia una mejor distribución de los asimilados para sustentar el crecimiento de los diferentes órganos. Con relación a este comportamiento, Luiz (1999) establece que la eficiencia de la fotosíntesis y de la conversión de los asimilados en biomasa es mayor cuando la temperatura se encuentra lo más cercana posible al óptimo recomendado para el cultivo.

En igual sentido, Luo (2006), Azcón y Talón (2008) y Marcelis y De Pascale (2009) plantean que un aumento de la temperatura, de la radiación solar o de la concentración de $\mathrm{CO}_{2}$, por encima o por debajo del límite óptimo que se establece para el tomate va en detrimento de la producción de asimilados que siguen el camino de la biosíntesis de las sustancias

Tabla 3. Respuesta de las diferentes variables evaluadas en el cultivo protegido del tomate (híbrido HA 3019) en dos épocas de plantación.

\begin{tabular}{|c|c|c|c|}
\hline Variables & Primavera-verano & Invierno & Esx y significación \\
\hline MST (final de la fase II) (g/m²) & 206,53 & 249,53 & $2.391 * * *$ \\
\hline MST (final de la fase III) $\left(\mathrm{g} / \mathrm{m}^{2}\right)$ & 598,93 & 706,26 & $4.568 * * *$ \\
\hline MST (final de la fase IV) $\left(\mathrm{g} / \mathrm{m}^{2}\right)$ & 821,23 & $1.002,04$ & $9.814 * * *$ \\
\hline Absorción de $\mathrm{N}\left(\mathrm{g} / \mathrm{m}^{2}\right)$ & 20,33 & 27,32 & $0,113^{* *}$ \\
\hline Absorción de P (g/m²) & 3,36 & 3,52 & $0,021 \mathrm{~ns}$ \\
\hline Absorción de K (g/m²) & 32,54 & 48,33 & $0,396 * * *$ \\
\hline Absorción de $\mathrm{Ca}\left(\mathrm{g} / \mathrm{m}^{2}\right)$ & 26,50 & 30,30 & $1,236^{* *}$ \\
\hline Absorción de $\mathrm{Mg}\left(\mathrm{g} / \mathrm{m}^{2}\right)$ & 2,51 & 3,66 & $0,028 * *$ \\
\hline Rend. calidad extra (t/ha) & 34,76 & 41,87 & $1,860 * *$ \\
\hline Rend. primera (t/ha) & 22,06 & 33,38 & $1,557 * *$ \\
\hline Rend. calidad E + P (t/ha) & 56,82 & 75,25 & $1,294 * * *$ \\
\hline Rend. calidad segunda (t/ha) & 6,12 & 6,24 & $0,498^{*}$ \\
\hline Rend. total (t/ha) & 62,94 & 81,49 & $1,288 * * *$ \\
\hline
\end{tabular}

MST: Masa seca total, Rend.: Rendimiento, E + P: extra + primera.

ns: No existieron diferencias significativas entre épocas de estudio para $\mathrm{P} \leq 0,05$.

$*$, ** y ***: Existieron diferencias significativas entre las épocas de estudio para $\mathrm{P} \leq 0,05, \mathrm{P} \leq 0,01$ y $\mathrm{P} \leq 0,001$, respectivamente. 
orgánicas necesarias para el mantenimiento del crecimiento, pues estos factores de clima influyen en el balance que se establece entre la pérdida de $\mathrm{CO}_{2}$ y la formación de estructuras celulares.

De igual forma, He y Zhang (2006) al aplicar un modelo de simulación matemática en el cultivo del tomate encontraron que el diámetro, la masa promedio del fruto y el rendimiento muestran una relación negativa con el incremento de la temperatura. Por su parte, Rodríguez y Gómez (2005) al cultivar diferentes híbridos de tomate en época de verano, con temperaturas máximas promedio de $39,60^{\circ} \mathrm{C}$, encontraron que los caracteres productivos evaluados decrecen con relación a la época de invierno, siendo el rendimiento, la fructificación y el peso de los frutos las variables que registraron los mayores porcentajes de reducción. De igual forma Quesada y Bertsch (2012) plantean que varios factores influyeron para no poder lograr un rendimiento superior en un estudio de estrategias de fertirriego en el cultivo protegido del tomate; el factor determinante fue las altas temperaturas que se registraron en el interior del invernadero. Según Gómez et al. (2000), los múltiples procesos que incluye una polinización eficiente son todos termosensibles.

El pH de la solución fertilizante estuvo entre 6,23-6,32 (Figura 1), mientras que en la solución del suelo fue de 7,00-7,19, ambos valores se consideran adecuados según lo establecido por Alarcón (2000), quien plantea que la correcta neutralización del ión $\mathrm{HCO}_{3}{ }^{-}$presente en el agua de riego permitirá obtener un pH a la salida del gotero de 5,50-6,50 y entre 6,00-7,20 en la solución nutriente en contacto con las raíces.

Los valores de $\mathrm{pH}$ de la solución del suelo fueron inferiores a los del análisis químico de suelo
(7,75 en $\mathrm{H} 2 \mathrm{O})$, lo que indica que la utilización de ácidos para neutralizar los bicarbonatos del agua de riego constituye un aspecto de manejo nutrimental necesario para mitigar el efecto desfavorable de un incremento del $\mathrm{pH}$ en el desarrollo de los cultivos, al garantizar el crecimiento de las raíces en un bulbo húmedo con un $\mathrm{pH}$ adecuado para la absorción de los nutrientes.

Durante la época primavera-verano, la concentración de $\mathrm{NO}_{3}{ }^{-}$en la solución del suelo tiende a ser superior, entre 109,96-130,54\%, a la determinada en el emisor en todas las fases del cultivo, mientras que en la época de invierno solo se acumula en la fase II (Figura 2). En el aumento del $\mathrm{NO}_{3}{ }^{-}$de la solución del suelo, en la época primaveraverano, pudo incidir procesos de mineralización de la materia orgánica, los cuales se ven favorecidos por las altas temperaturas y un régimen de humedad favorable; condiciones que caracterizan el ambiente de una casa de cultivo (Hernández et al., 2007). Por otra parte, hay que tener en cuenta que en la época de invierno se cuantificaron valores superiores de biomasa, rendimiento y extracción de nutrientes, factores que pudieron condicionar la disminución del $\mathrm{NO}_{3}{ }^{-}$en la solución del suelo para esta época de estudio.

La movilidad del $\mathrm{NO}_{3}{ }^{-}$en el suelo constituye otro de los factores que pudo incidir en las variaciones determinadas. Este anión se considera muy móvil $y$, una vez en el suelo, no entra en las reacciones de intercambio que se producen con el complejo absorbente del suelo, por lo que inmediatamente después de su aplicación estará disponible para las plantas. Lo anterior pudo traer como consecuencia que en el momento del muestreo ya la planta había consumido parte del $\mathrm{NO}_{3}{ }^{-}$aplicado y que su

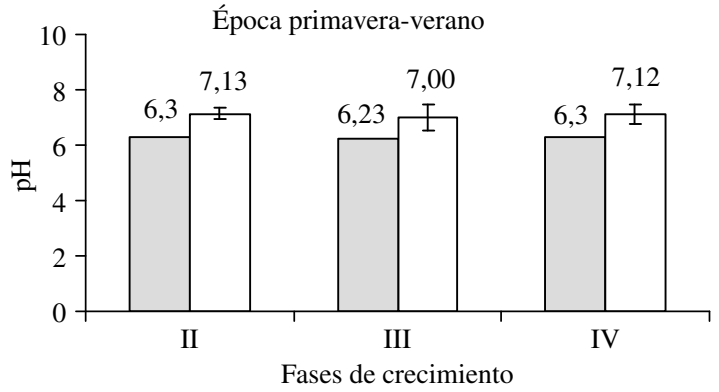

Solución fertilizante

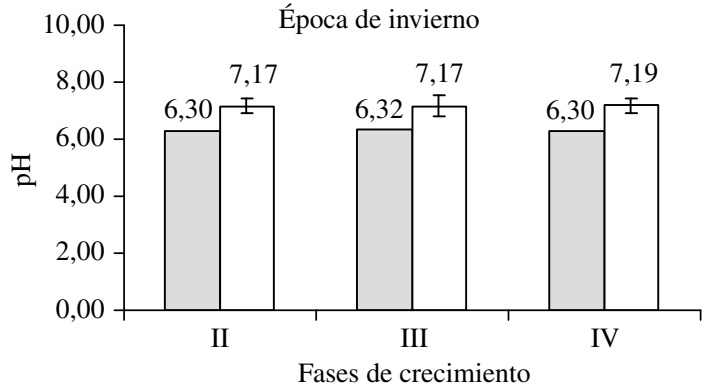

Solución del suelo

Figura 1. Valores medios de $\mathrm{pH}$ cuantificados en la solución fertilizante y en la solución del suelo para las fases II $(\mathrm{n}=7)$, III $(n=6)$ y IV $(n=10)$ del ciclo de crecimiento en el cultivo protegido del tomate, híbrido HA 3019. 


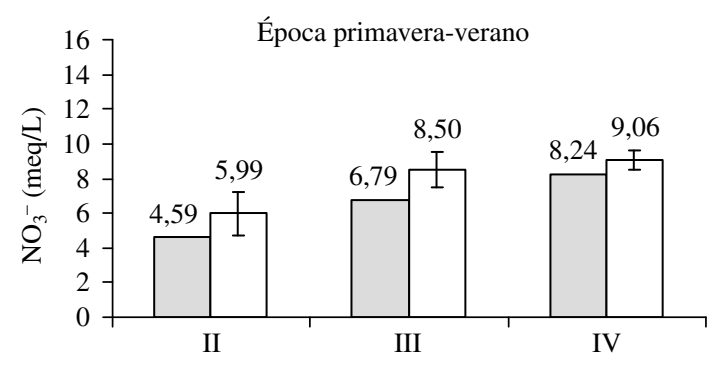

Fases de crecimiento

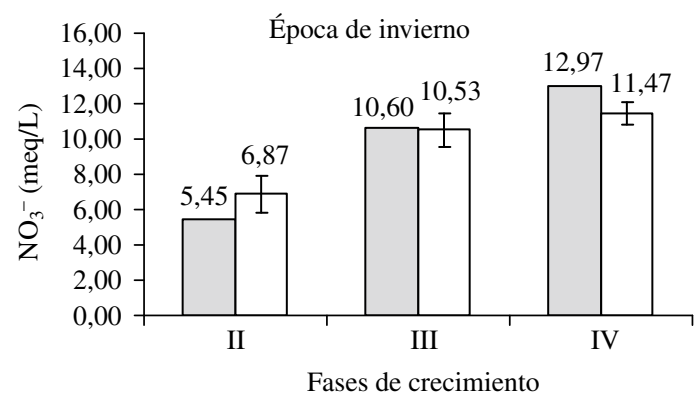

Solución del suelo

Figura 2. Valores medios de $\mathrm{NO}_{3}{ }^{-}$(meq/L) cuantificados en la solución fertilizante y en la solución del suelo para las fases II $(\mathrm{n}=7)$, III $(\mathrm{n}=6)$ y IV $(\mathrm{n}=10)$ del ciclo de crecimiento en el cultivo protegido del tomate, híbrido HA 3019.

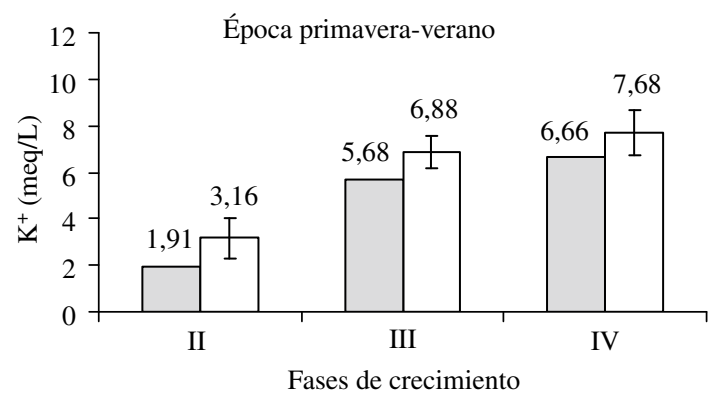

Solución fertilizante

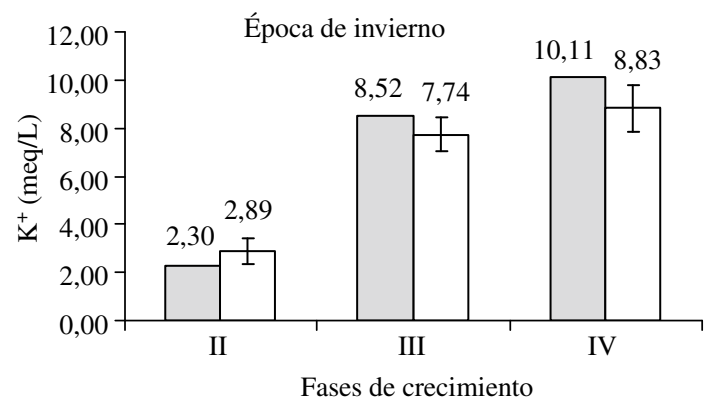

Solución del suelo

Figura 3. Valores medios de $\mathrm{K}^{+}$(meq/L) cuantificados en la solución fertilizante y en la solución del suelo para las fases II $(\mathrm{n}=7)$, III $(n=6)$ y IV $(n=10)$ del ciclo de crecimiento en el cultivo protegido del tomate, híbrido HA 3019.

concentración en la solución del suelo disminuyera en las fases y épocas de mayor crecimiento.

Con relación a este anión, Casas (1999) plantea que el $\mathrm{NO}_{3}{ }^{-}$en la solución del suelo puede oscilar entre 75 y $125 \%$ en función de la solución de entrada, mientras que Lao et al. (2004) encontraron, en condiciones de invernadero, que el $\mathrm{NO}_{3}{ }^{-}$se acumula en las fases de crecimiento vegetativo, mientras que en las etapas de plena producción las concentraciones se igualan, con períodos donde los valores en la solución del suelo son inferiores a la solución fertilizante.

En sistemas de producción hortícola intensiva, se ha recomendado el empleo de sondas de succión para optimizar el manejo de la fertilización nitrogenada, lo que permite conocer de forma rápida, durante el desarrollo del cultivo, si el programa de fertilización nitrogenada que se está llevando a cabo es el adecuado. En este sentido, Thompson et al. (2006) señalan que es necesario el monitoreo del $\mathrm{NO}_{3}{ }^{-}$con el objetivo de evitar el exceso y la posible contaminación producida por nitratos de origen agrario. Por su parte Cano y Rojo (2004) plantean que no solo es importante diagnosticar un exceso de $\mathrm{N}$ en suelo, pues una baja concentración puede ser indicativo no solo de una deficiencia de $\mathrm{N}$, sino también de un excesivo régimen de riego con peligros de lixiviación y contaminación de las aguas subterráneas.

La concentración de $\mathrm{K}^{+}$mostró un comportamiento similar al del $\mathrm{NO}_{3}^{-}$, con una tendencia a acumularse en la solución del suelo en todas las fases del cultivo durante la época primavera-verano, y solo en la fase II de la época de invierno (Figura 3). Los menores porcentajes de variación se cuantificaron en las fases III y IV, respuesta que se corresponde con las exigencias del cultivo, pues el tomate requiere de grandes cantidades de $\mathrm{K}$ en los períodos de llenado del fruto y de cosecha, necesarias para sustentar la formación del rendimiento. Esta alta demanda del cultivo pudo traer como consecuencia que el $\mathrm{K}$ aplicado en solución nutriente fuera rápidamente absorbido por la planta y que los contenidos variaran entre la fertirrigación y la toma de la muestra, lo que 
se encuentra en correspondencia con lo planteado por Guzmán (2006), quien establece como uno de los problemas en el control y monitoreo de los nutrientes en la solución del suelo o del sustrato, la rápida movilidad de algunos iones. Este autor clasifica al $\mathrm{K}^{+}$como un elemento de absorción rápida, conjuntamente con el $\mathrm{NO}_{3}^{-}$, el $\mathrm{NH}_{4}^{+}$y el $\mathrm{H}_{2} \mathrm{PO}_{4}^{-}$, los cuales pueden ser removidos de la solución en pocas horas.

Casas (1999) y Lao et al. (2004) establecen, tanto para el cultivo de tomate en suelo como en sustrato, que los valores de $\mathrm{K}^{+}$en la solución del suelo pueden variar entre 50 y $100 \%$ con relación a la solución fertilizante, y su comportamiento depende de la alta demanda que de este nutriente ejercen los cultivos de alto rendimiento, de su rápida absorción, del balance que existe entre los procesos vegetativos y generativos y de las propiedades físico-químicas del suelo.

La concentración de $\mathrm{Ca}^{++}$en la solución del suelo fue siempre superior a la cuantificada en el gotero para las dos épocas de estudio (Figura 4). Sin embargo, teniendo en cuenta los altos contenidos de $\mathrm{Ca}^{++}$intercambiable que presenta este suelo, podría esperarse una mayor concentración del elemento en la solución del suelo, pues según Triana (2001), de la composición de los cationes adsorbidos depende la composición de la solución del suelo. Si el suelo contiene una mayor proporción de $\mathrm{Ca}^{++}$adsorbido, entonces al aplicar fertilizantes solubles se desplazará a la solución del suelo principalmente $\mathrm{Ca}^{++}$.

Las altas absorciones de Ca que realiza el cultivo, segundo elemento que en mayor medida extrajo la planta, influyeron en que las variaciones cuantificadas entre la solución del suelo y la solución fertilizante no fueran tan amplias como las indicadas por Lao et al. (2004), quienes establecen porcentajes de variación promedio de $160 \%$. Los porcentajes de variación del $\mathrm{Ca}^{++}$correspondientes a la época de invierno tendieron a ser inferiores a los determinados en la época primavera-verano, lo que pudo estar condicionado no solo por indicadores superiores de crecimiento y producción en el invierno, sino también por las variables de clima que caracterizaron cada época de estudio.

En las fases III y IV de la época primavera-verano se presentaron temperaturas superiores en el interior de la instalación con relación a la época de invierno, y sobre este aspecto Lezcano (2004) plantea que en condiciones de alta temperatura y evapotranspiración el crecimiento del fruto es muy intenso, sin embargo la movilidad del $\mathrm{Ca}^{++}$en la planta es lenta, por lo que la cantidad de $\mathrm{Ca}^{++}$que llega al fruto no es suficiente para cubrir la demanda nutricional en cultivares de alto rendimiento, con una tendencia a acumularse en la planta o en el suelo. A esto se une que el $\mathrm{Ca}^{++}$, al igual que el $\mathrm{Mg}^{++}$, es absorbido por la planta a una velocidad menor que la del transporte por el flujo de agua, por lo que tienden a acumularse en la superficie de la raíz. Esta particularidad, unida a que la planta realiza una escasa absorción del elemento pudo incidir en que el $\mathrm{Mg}^{++}$se acumulara también en la solución del suelo (Figura 5); resultados que coinciden con los obtenidos por Triana (2001) y Lao et al. (2004), quienes calcularon un incremento del $\mathrm{Mg}^{++}$en torno a la raíz de hasta $210 \%$.

Uno de los aspectos más importantes en el manejo de la solución nutriente es lograr un adecuado balance entre los cationes $\mathrm{K}^{+}, \mathrm{Ca}^{++} \mathrm{y} \mathrm{Mg}^{++}$. De esta forma Steiner (1984) elaboró para tomate una composición estándar de la solución nutritiva que presenta una relación $\mathrm{K}^{+}: \mathrm{Ca}^{++}: \mathrm{Mg}^{++}$entre

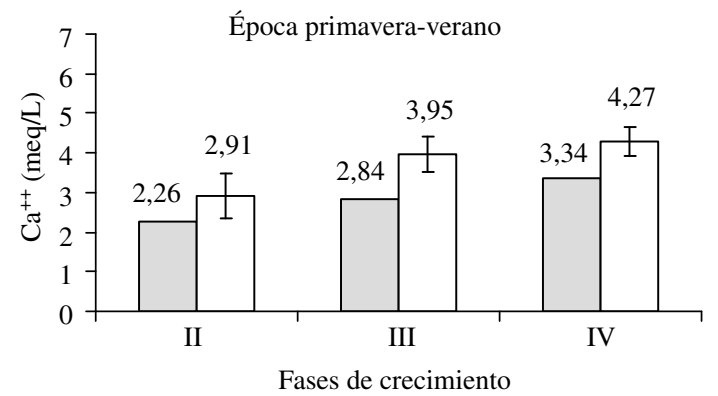

Solución fertilizante

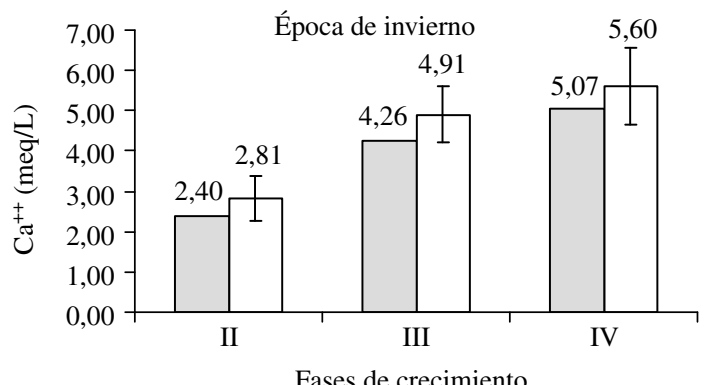

Fases de crecimiento

Solución del suelo

Figura 4. Valores medios de $\mathrm{Ca}^{++}(\mathrm{meq} / \mathrm{L})$ cuantificados en la solución fertilizante y en la solución del suelo para las fases II $(\mathrm{n}=7)$, III $(n=6)$ y IV $(n=10)$ del ciclo de crecimiento en el cultivo protegido del tomate, híbrido HA 3019. 


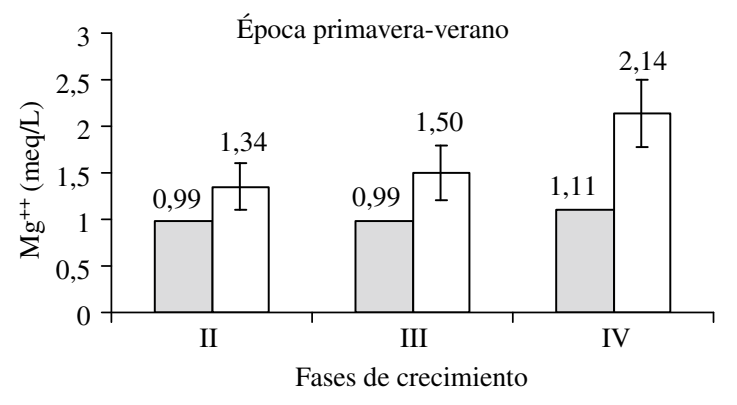

Solución fertilizante

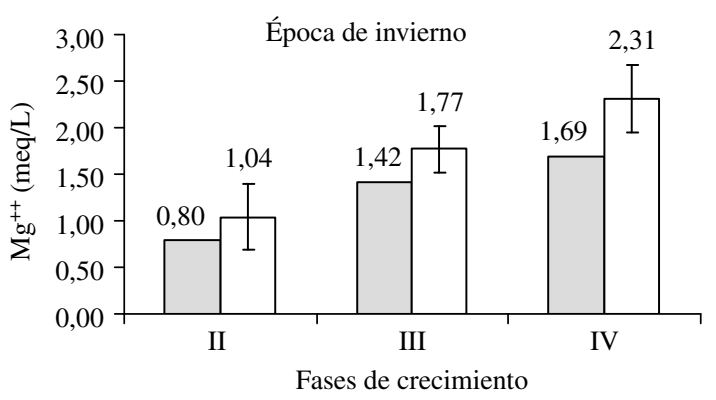

Solución del suelo

Figura 5: Valores medios de $\mathrm{Mg}^{++}$(meq/L) cuantificados en la solución fertilizante y en la solución del suelo para las fases II $(\mathrm{n}=7)$, III $(\mathrm{n}=6)$ y IV $(\mathrm{n}=10)$ del ciclo de crecimiento en el cultivo protegido del tomate, híbrido HA 3019.

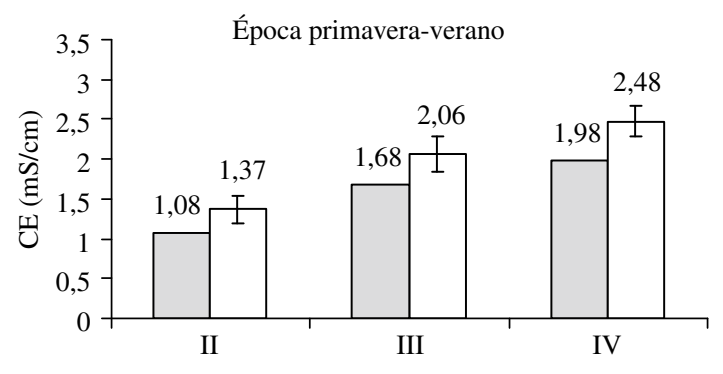

Fases de crecimiento

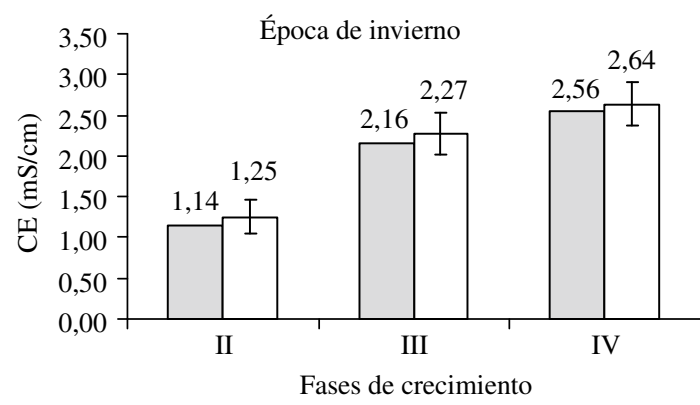

Solución del suelo

Figura 6: Valores medios de CE $(\mathrm{mS} / \mathrm{cm})$ cuantificados en la solución fertilizante y en la solución del suelo para las fases II $(\mathrm{n}=7)$, III $(n=6)$ y IV $(n=10)$ del ciclo de crecimiento en el cultivo protegido del tomate, híbrido HA 3019.

(40-70):(25-45):(5-16) (\% del total en meq/L), válida también para la solución del suelo. Esta relación se cumple para los valores de la solución del suelo que se analizan en el presente acápite (50,97:36,39:13,84 para primavera-verano y 49,75:36,39:13,84 para invierno), lo que indica que el cultivo recibió, en ambas épocas, una solución nutritiva que le permitió a la planta disponer de una solución del suelo con un balance apropiado entre estos cationes, aun cuando en el suelo se presentaron relaciones inadecuadas entre ellos. Esto sugiere que el empleo de soluciones fertilizantes equilibradas constituye un aspecto importante para mitigar limitaciones que presenten los suelos en estudio.

La CE promedio en la solución del suelo tendió a ser superior a la determinada en el emisor, con porcentajes que variaron entre 122,72-126,59\% (época primavera-verano) y 103,05-109,50\% (época de invierno) (Figura 6). Por tal motivo, con las soluciones nutritivas propuestas para cada época de estudio se logra efectuar una fertirrigación correcta si se tiene en cuenta que la CE en la solución del suelo puede ser superior a la cuantificada en el gotero hasta $1,50 \mathrm{mS} / \mathrm{cm}$, según lo que establecen Alarcón (2000) e Imas (2002); una CE en la solución del suelo inferior a la emitida por el gotero muestra la necesidad de aumentar el aporte de nutrientes.

Por otra parte, es de destacar que la variación máxima obtenida para la $\mathrm{CE}$ en sondas de succión fue de $0,50 \mathrm{mS} / \mathrm{cm}$ (Fase IV en invierno) con relación a la solución fertilizante, lo que indica bajo peligro de salinización en el suelo. En este sentido, Segura y Martínez (1990) establecen que la CE es una expresión de la cantidad total de sales disueltas en una determinada solución nutritiva y que a medida que los valores son más altos se incrementa el riesgo de salinización y contaminación, con efectos fisiológicos, morfológicos y bioquímicos negativos, una disminución de la fotosíntesis y afectaciones en el proceso de floración y fructificación. El principal efecto de la CE se relaciona con el factor osmótico y una mayor dificultad de la planta para absorber agua, con un gasto de energía que puede ser en detrimento de la energía metabólica y de los 
compuestos orgánicos que se destinan a los diferentes órganos de la planta en crecimiento (Munns, 2005; Goycovic y Saavedra, 2007).

Los valores cuantificados de $\mathrm{pH}, \mathrm{CE}$ y concentración de aniones y cationes en la solución del suelo y los porcentajes de variación con relación a la solución fertilizante pueden utilizarse como referencia para el manejo racional del fertirriego en el cultivo protegido del tomate en condiciones de suelo y clima (época) similares a las del presente estudio. Lo que se sustenta en que las soluciones nutritivas donde se efectuaron los muestreos a partir de sondas de succión permitieron obtener resultados productivos favorables en cada época de estudio, en correspondencia con las metas identificadas para el cultivo protegido del tomate en Cuba.

No obstante, el análisis de la solución del suelo no puede considerarse como único método de diagnóstico para predecir alteraciones nutricionales en el cultivo protegido del tomate, pues otros factores influyen en la acumulación de nutrientes en torno a la raíz, a este aspecto debe integrarse el monitoreo del clima, del suelo, del estado nutricional y sanitario de la plantación y de la calidad del agua de riego, así como el conocimiento de las características del cultivar utilizado, que unidos a un adecuado manejo agronómico posibilitará obtener los beneficios deseados.

\section{Conclusiones}

1. La masa seca, la absorción de N, P, K, Ca y Mg y el rendimiento en el cultivo protegido del tomate en época de invierno fue significativamente superior a la obtenida en primavera-verano.

2. La alta movilidad del $\mathrm{NO}_{3}^{-}$y del $\mathrm{K}^{+}$y la presencia de indicadores productivos y de crecimiento superiores en la época de invierno, así como en las fases de llenado del fruto y de cosecha pueden condicionar su disminución en la solución del suelo, mientras que el $\mathrm{Ca}^{++}$y el $\mathrm{Mg}^{++}$se acumulan siempre en el bulbo húmedo.

3. La concentración de $\mathrm{NO}_{3}^{-}$en la solución del suelo estuvo entre 5,99-9,06 meq/L y 6,87-11,47 $\mathrm{meq} / \mathrm{L}$ para las épocas de primavera-verano e invierno, respectivamente, mientras que el potasio varió entre 3,16-7,67 meq/L y 2,89-8,83 meq/L. La concentración de $\mathrm{Ca}^{++}$en la solución del suelo fue de 2,91-4,27 meq/L y 2,81-5,60 $\mathrm{meq} / \mathrm{L}$ para las épocas de primavera-verano e invierno, respectivamente, mientras que el $\mathrm{Mg}^{++}$ varió entre 1,34-2,14 meq/L y 1,04-2,31 meq/L.

4. La variación máxima obtenida para la $\mathrm{CE}$ en sondas de succión con relación a la solución fertilizante fue de $0,50 \mathrm{mS} / \mathrm{cm}$ en la Fase IV del invierno, lo que indica bajo peligro de salinización en el suelo.

\section{Literatura Citada}

Alarcón, A. L.

2000. Tecnologías para cultivos de alto rendimiento. $1^{\text {a Edición. }}$ Novedades Agrícolas S.A. España, 384 pp.

Azcón, J.; M. Talón.

2008. Fundamentos de fisiología vegetal. $2^{\text {a }}$ Edición. Ediciones Universidad de Barcelona. Barcelona-España. 656 pp.

Cadahía, C.

2005. Fertirrigación de cultivos hortícolas, frutales y ornamentales. $3^{\text {a }}$ Edición. Mundi Prensa. Madrid-España. $475 \mathrm{pp}$.

Cano, D.; J. Rojo.

2004 Correlación de medidas obtenidas a partir de sondas de succión y extracto de saturación del suelo regado con aguas salinas. Ingeniería del agua, 11 (3): 329-338.

Casanova, A.; Cardoza, H.; Hernández, M.; Gómez, O.; Pupo, F.; Chailloux, M.; Depestre, T.; Moreno, V.; Hernández, J.C. 2007. Manual para la producción protegida de hortalizas. $2^{\text {a }}$ Edición. Editora Liliana. La Habana-Cuba. 125 pp.

Casas, A.

1999. Solución nutritiva, principios básicos, comportamiento e interacción. En: Cultivos sin Suelos II. 2a Edición. Fernández, M.; Cuadrado I.M. FIAPA. Almería-España, pp. 229-247.
Cuba, MINAG.

2010. Especificaciones de calidad para la compra-venta de productos agrícolas con destino a su comercialización para el consumo. $1^{\text {a }}$ Edición. Agrinfor. Ciudad de La Habana: Cuba. 47 pp.

Duarte, C.; Ajete, M.; González, F.; Bonet, C.; Sierra, L.O. 2010. Dosificación de fertilizante para el fertirriego del tomate protegido en Ciego de Ávila. Revista Ciencias Técnicas Agropecuarias, 19 (3): 25-29.

Goykovic, C.; Saavedra V.

2007. Algunos efectos de la salinidad en el cultivo del tomate y prácticas agronómicas de su manejo. Idesia, 25 (3): 47-58.

Gómez, O.; Casanova, A.; Laterrot, H.; Anais, G.

2000. Mejora genética y manejo del cultivo del tomate para la producción en el Caribe. $1^{\text {a }}$ Edición. Editora Liliana. La Habana-Cuba. 159 pp.

Granados, M.R.; Thompson, R.B.; Fernández, M.D.; Gallardo, M.; Gázquez, J.C.

Uso de sondas de succión para el manejo de la fertilización nitrogenada en un cultivo de tomate bajo condiciones de invernadero. http://www.publicacionescajamar.es/. Consultado 1/7/2013. 
Guzmán, J.M.

2006. Reutilización y recirculación: eficiencia y calidad de los sistemas cerrados. En: Avances sobre fertirriego en la floricultura colombiana. $1^{\text {a }}$ Edición. Florez V.J.; Fernández, A.; Miranda, D.; Chaves, B.; Guzmán, J.M. Universidad Nacional de Colombia. Bogotá-Colombia. Pp. 479-499.

He, C.; Zhang, Z.

2006. Modeling the relationship between tomato fruit growth and the effective accumulated temperature in solar greenhouse. Acta Horticulturae, 718: 581-588.

Hernández, M.I.; Chailloux, M.; Moreno, V.; Ojeda, A.; Salgado, J.M.; Bruzón, O.

2009. Relaciones nitrógeno-potasio en fertirriego para el cultivo protegido del tomate en suelo Ferralítico Rojo. Pesquisa Agropecuaria Brasileña, 44 (5): 429-436.

Hernández, A.; Morales, M.; Morrel, F.; Borges, Y.; Moreno,

I.; Ríos, H.; Vargas, D.

2007. Algunos resultados sobre las pérdidas de carbono en ecosistemas con suelos Ferralíticos Rojos lixiviados en Clima tropical subhúmedo de Cuba. Cultivos Tropicales, 28 (3): 55-60.

Imas, $\mathrm{P}$.

Manejo de Nutrientes por Fertirriego en Sistemas Frutihortícolas. http://www.ipipotash.org/ Consultado: octubre 22/10/2002.

Lao, M.T.; Jiménez, S.; Eymar, E.; Fernández, E.J.

2003. Determination of spatial variability of nutrient composition of soil solutions in greenhouses by using suction cups. Soil Science and Plant Analysis, 34 (5 \& 6): 865-879.

Lao, M.T.; Jiménez, S.; Eymar, E.; Fernández, E.J.

2004. Nutrient levels of the soil solution obtained by means of suction cups in intensive tomato cultivation. Revista Internacional de Botánica Experimental (Phyton), 73: 19-37.

Lezcano, I.

Las temperaturas altas y la deficiencia de calcio en tomate. http://www.inpofos.org/ppiweb/iamex.nsf/. Consultado: 23/9/2007.

Luiz, J.

1999. Fisiología de los cultivos protegidos. $1^{\text {a }}$ Edición. UFSM. Brasil, 142 pp.

Luo, W.

2006. Predicting leaf area of three greenhouse crops using PAR and temperature. Acta Horticulturae, 718: 589-598.

Marcelis, L.F.; De Pascale, S.

2009. Crop management in greenhouses: adapting the growth conditions to the plant needs or adapting the plant to the growth conditions? Acta Horticulturae, (807): 97-102.
Munns, R.

2005. Genes and salt tolerance: ringing them together. New Phytologist, 167 (3): 645-660.

Murillo, C.A.; Tafur, H.; Floréz, V.J.; Burbano, R.

2006. Modelación del movimiento del agua en el suelo. En: $1^{a}$ Edición. Florez, V.J.; Fernández, A.; Miranda, D.; Chaves, B.; Guzmán, J.M. Universidad Nacional de Colombia. Bogotá-Colombia. Pp. 359-375.

Quesada, G.; Bertsch, F.

2012. Fertirriego en el rendimiento de híbridos de tomate producidos en invernadero. Agronomía Mesoamericana, 23 (1): 1-11.

Rodríguez, G.; Gómez, O.

2005. Evaluación de híbridos F1 adaptados al sistema de cultivo protegido. Temas de Ciencia y Tecnología, 9 (25): 7-12.

Romo, M.

2006. Tendencias tecnológicas en la fertirrigación de cultivos de flores en Colombia. En: $1^{\text {a }}$ Edición. Florez, V.J.; Fernández, A.; Miranda, D.; Chaves, B.; Guzmán, J.M. Universidad Nacional de Colombia. Bogotá-Colombia. Pp. 377-406.

Salas, M.C.; Vargas, J.B.; Sánchez-Romer, J.

2009. Using suction cup to improve the monitoring of soil solution in a greenhouse fertigated crop. Acta Horticulturae, 807: 301-306.

Segura, M.L.; Martínez, M.

1990. Nutrición del cultivo del tomate a través del riego por goteo. Agrícola Vergel, 180 (9): 920-925.

Steiner, A.A.

1966. The influence of the chemical composition of nutrient solutions on the production of tomato plant. Plant and Soil, 24 (2): 454-466.

Thompson, R.B., Martínez, C., Fernández, M.D., López-Toral, J.R., Gallardo, M.; Giménez, C.

2006. Management factors contributing to nitrate leaching loss from a greenhouse-based intensive vegetable production system. Acta Horticulturae, 700: 179-184

Triana, M. P.

2001. La importancia de los elementos secundarios (Ca, $\mathrm{Mg}$ y S) en la nutrición de las plantas. En: Los elementos secundarios $(\mathrm{Ca}, \mathrm{Mg}$ y S) en la agricultura. 1ra. Edición. Silva-Mojica, F. Sociedad Colombiana de la Ciencia del Suelo. Bogotá-Colombia, p. 1-13.

Voogt, W.

2006. Evaluation of the fertigation model, a decision support system for water and nutrient supply for soil grown greenhouse crops. Acta Horticulturae (ISHS), 718: 531-538. 